\title{
Repression of miR-135b-5p promotes metastasis of early-stage breast cancer by regulating downstream target SDCBP
}

\author{
Tianjie $\mathrm{Pu}^{1,2} \cdot$ Mengjia Shen ${ }^{1,2} \cdot$ Shi $\mathrm{Li}^{3} \cdot$ Libo Yang $^{1,2} \cdot$ Hongwei Gao $^{2} \cdot$ Lin Xiao $^{2} \cdot$ Xiaorong Zhong $^{4,5}$. \\ Hong Zheng ${ }^{4,5} \cdot$ Yueping Liu $^{3} \cdot$ Feng $\mathrm{Ye}^{2,5,6} \cdot$ Hong Bu $\mathrm{Bu}^{1,2,5,6}$
}

Received: 17 October 2018 / Revised: 8 April 2019 / Accepted: 9 April 2019 / Published online: 25 April 2019

(c) United States \& Canadian Academy of Pathology 2019

\begin{abstract}
Metastasis is an essential event for breast cancer (BC) progression even after initial surgery. The identification of patients with a high probability of metastasis at an early stage is particularly important in clinical practice and requires individualized treatment or early prevention. A retrospective study of 242 cases of ductal carcinoma in situ with microinvasion (DCIS-Mi), the first stage of invasive BC, was performed in this follow-up analysis. Of all patients, 8 developed metastases, and they were all included for further mechanistic studies with control group of 24 DCIS-Mi by matched-pair designing. By screening DCIS-Mi with different prognoses, we found that the DCIS-Mi that metastasized had significantly lower miR-135b-5p expression than the DCIS-Mi that did not. The function of miR-135b-5p was studied in vitro and in vivo invasion and metastasis assays. We also validated a novel target gene for miR-135b-5p, syndecan binding protein (SDCBP), and assessed the functional consequences of SDCBP by invasion assays. By checking different BC cell lines, a strong inverse correlation between miR-135b-5p and SDCBP expression was recorded. For the functional study, the inhibition of miR-135b-5p was accompanied by increased BC cell growth, epithelial-mesenchymal transition (EMT), migration and invasion in vitro. Interestingly, silencing SDCBP can reverse miR-135b-5p-dependent EMT and proliferation. In vivo studies demonstrated that the newly revealed miR-135b-5p/SDCBP axis increased cell proliferation, invasion and malignant transformation, as well as promoted metastasis in a xenograft tumor mouse model. Thus, our clinical patient cohort and functional data suggest that miR-135b-5p/SDCBP is a crucial determinant of BC metastasis at a very early stage. Our results may shed light on the importance of miR-135b-5p molecular diagnosis and prognosis, as well as the early prevention of $\mathrm{BC}$ for metastasis.
\end{abstract}

Supplementary information The online version of this article (https:// doi.org/10.1038/s41374-019-0258-1) contains supplementary material, which is available to authorized users.

$\triangle$ Feng Ye

fengye@scu.edu.cn

1 Department of Pathology, West China Hospital, Sichuan University, Chengdu, China

2 Laboratory of Pathology, West China Hospital, Sichuan University, Chengdu, China

3 Department of Pathology, The Fourth Hospital of Hebei Medical University, Shijiazhuang, China

4 Laboratory of Molecular Diagnosis of Cancer \& Cancer Center, West China Hospital, Sichuan University, Chengdu, China

5 Breast Disease Center, West China Hospital, Sichuan University, Chengdu, China

6 Key Laboratory of Transplant Engineering and Immunology, Ministry of Health, West China. Hospital, Sichuan University, Chengdu, China

\section{Introduction}

Breast cancer (BC) is one of the most common malignant tumors diagnosed among women, serving as a leading cause of cancer-related death worldwide. Due to the wide application of general health examination and advanced diagnostic technology, the detection rate of early $\mathrm{BC}$ has increased tremendously in recent years, especially for the early diagnosis of ductal carcinoma in situ (DCIS) and microinvasive carcinoma (DCIS-Mi). DCIS with or without microinvasion accounts for approximately 20 to $25 \%$ of newly diagnosed BC in Europe and the United States [1, 2] and $10-15 \%$ in China [3]. DCIS-Mi is defined by the American Joint Committee on Cancer (AJCC 8th edition) [4], as DCIS with no invasive foci $>1 \mathrm{~mm}$, representing the first stage of invasive BC.

Although patients with DCIS-Mi often have a favorable prognosis, some patients still experience recurrence and metastasis. Previously, several groups have reported the 
prognostic impact of age, tumor size, nuclear grade, lymph node metastasis and other factors on DCIS-Mi patients, but there remain many controversial questions about the biological behavior and prognosis [5-10]. Our previous study retrospectively reviewed 242 cases of DCIS-Mi, 280 cases of pure DCIS, and 347 cases of IDC-T1. We found out that high-grade DCIS and DCIS-Mi had similar clinical characteristics, but their prognoses were quite different [11]. Traditional pathological morphology is insufficient for predicting the prognosis of early invasive BC. Thus, molecular markers that identify cells with metastatic potential and differentiate the patients for whom the tumor will metastasize at an early stage will help precision individualized treatment and early prevention.

Many multigene tests have been used to assist the diagnosis and prognosis of BC, such as MammaPrint, Oncotype $\mathrm{Dx}^{\circ}$ and PAM50. Prediction models for either DCIS or DCIS-Mi have been scarcely studied. In BC, miRNAs have been shown to regulate cell cycle progression, apoptosis, angiogenesis, epithelial-mesenchymal transition (EMT), tumor microenvironment, migration, invasion, metastasis, and drug resistance, as well as the differentiation and self-renewal of BC stem cells. As metastatic BC is the most advanced and difficult to handle, fully understanding molecules promoting metastasis will help with precision molecular diagnosis and early prevention. MiRNAs are very stable even in formalin-fixed paraffin-embedded samples, which makes them potential biomarkers for cancer prognosis and therapy prediction, especially after long-term follow-up observation [12-15]. The role of miRNAs in prognosis studies for DCIS-Mi has been scarcely studied, especially with up to 5-10 years following up.

In this study, microarray and qRT-PCR were used to screen for metastasis-related miRNAs. MiR-135b-5p was isolated as an essential biomarker to predict $\mathrm{BC}$ metastasis at a very early stage, e.g., DCIS-Mi. Our clinical patient cohort and functional data suggested that the inhibition of miR-135b-5p could promote metastasis by downregulating the direct target SDCBP. Our results may shed light on the importance of miR-135b-5p molecular diagnosis and prognosis, as well as the early prevention of $\mathrm{BC}$ metastasis.

\section{Materials and methods}

\section{Clinical specimen collection}

A retrospective observational study of 242 cases of DCIS-Mi, 280 cases of pure DCIS, and 347 cases of invasive ductal carcinoma (IDC)-T1 from 1997 to 2014 in the West China Hospital was designed [11]. We refer to The College of American Pathologists (CAP) DCIS protocol, as follows: (1)
Up to $2 \mathrm{~cm}$ : The entire specimen should be examined and all tissues likely to be involved by DCIS should be complete submission. The specimen should be thinly sectioned by 0.2 $\mathrm{cm}$ and entirely submitted for microscopic evaluation. (2) $>2$ $\mathrm{cm}$ : We will selective sampling of large excisions or mastectomies to include areas likely to contain DCIS (e.g., tissue with radiologic calcifications or grossly abnormal tissue). Sectioned by $0.2 \mathrm{~cm}$ and microscopic examination is required. There is a possibility of undetected areas of invasion if the area involved by DCIS is not completely examined. In this case, we will perform lymph node sampling. A single H\&E section from each block is sufficient for routine evaluation. If additional studies are performed, these should be recommended (i.e., serial section at H\&E levels or immunohistochemical studies). After a median follow-up of 109 months, a total of 8 patients of all the DCIS-Mi developed metastasis which were included for following mechanism study with control group of 24 DCIS-Mi by matched-pair designing.

\section{RNA extraction and miRNA array assay}

The BC epithelial cells of tumor tissue were harvested by laser capture microdissection technology to avoid tumor heterogeneity. Microinvasion is defined by the AJCC as invasion measuring $1 \mathrm{~mm}$ or less in size. The microinvasive lesions may disappear in subsequent sections. So, we chose DCIS from cases with microinvasion instead of the individual microinvasive foci. Total RNA was isolated using TRIzol reagent according to the manufacturer's protocol (Invitrogen, USA). The microarray data of early BC formalin-fixed paraffin-embedded samples with different prognosis was provided by $\mathrm{Li}$ et al. [16]. For each miRNA, fold change was calculated, with $p<0.05$ or fold change $>$ 1.5 representing genes upregulated and fold change $\leq 0.5$ representing genes downregulated.

\section{RNA reverse transcription and real-time PCR}

The procedures of miRNA reverse-transcribed to cDNA and quantification were carried out according to the manual of the All-in-One ${ }^{\mathrm{TM}}$ miRNA qRT-PCR Detection Kit (GeneCopoeia, Guangzhou, China). U6, U44, and U47 transcripts as a normalization signal. Primers for all the miRNAs were purchased from GeneCopoeia (Supplementary Table 1). Levels of mature mRNAs were quantified by quantitative real-time PCR (qRT-PCR) using the SYBR Green Realtime PCR Master Mix (BioRad, USA). Primers for target genes were from Invitrogen (Invitrogen, USA), $\beta$-actin and GAPDH were used for normalization (Supplementary Table 2). Quantitative PCR was carried out using BioRad CFX96 system (Applied Biosystems, Hercules, USA). The relative amount of miRNA or mRNA in each sample was calculated using the comparative $\mathrm{C}_{\mathrm{T}}$ method. 


\section{Cell lines and cell culture}

Human BC cell lines MCF-7, SK-BR-3, and MDA-MB-231 were kindly provided by Dr. Hong Zheng (Laboratory of Molecular Diagnosis of Cancer \& Cancer center, Sichuan University). All cell lines had never been passaged longer than 3 months and the cell lines were characterized by Genetic Testing Biotechnology Corporation (Suzhou, China) using short tandem repeat markers.

\section{RNA oligonucleotides, plasmids, small interfering RNA, and transfection}

miR-135b-5p inhibitor, specific siRNAs were purchased from GenePharma (Shanghai, China). The cholesterolmodified antagomiR-135b-5p (anti-miR-135b-5p) and their respective negative control miRNAs were obtained from RiboBio (Guangzhou, China). The negative controls (inhibitor-NC, si-NC, and antagomiR-NC) consisting of random sequences had no detectable effects in human cell lines or tissues. The sequences of the siRNAs, miRNAs, and negative controls (NC) are listed in Supplementary Table 3. The coding domain sequence of human SDCBP mRNA was cloned into Lv201 plasmid (GeneCopoeia, Guangzhou, China). Cells cultured in 6-well plates were transfected with $100 \mathrm{nM}$ per well siRNA and miRNA inhibitor or with $200 \mathrm{nM}$ per well antagomiRNA using Lipofectamine 3000 (Life Technologies, USA) according to the manufacturer's protocol.

\section{Protein extraction and western blot analysis}

Proteins of cells were extracted with mammalian protein extraction kit (Pierce, Rockford, IL, USA) and protein concentrations were determined by the Bradford Assay (BioRad, USA). Protein lysates $(20 \mu \mathrm{g})$ were then resolved on SDS-PAGE and transferred to poly-vinylidene fluoride membranes (Millipore, Germany). The membranes were incubated with primary antibodies: CDH1 (1:1000, ZENBIO, China), Vim (1:2000, ZENBIO, China), Snail (1:1000, ZENBIO, China) and GAPDH (1:2000, ZENBIO, China).

\section{Cell proliferation, colony formation, apoptosis, migration, and invasion assays}

Cell proliferation was detected by the EnoGeneCell ${ }^{\mathrm{TM}}$ Counting Kit-8 (CCK-8) (Enogene, China) according to the manufacturer's protocol. In colony formation assay, cells were seeded into normal plates at a density of 1000 cells in six-well plates. After 2 weeks, the resulting colonies were stained with crystal violet and counted. Apoptosis was detected by fluorescein isothiocyanate (FITC)-Annexin V/7AAD double staining (4A Biotech, China) using flow cytometry. Wound-healing assay was applied to measure cell migration. Briefly, cells were grown as confluent monolayers in six-well plates. The cell monolayers were scratched with a $200 \mu$ l pipette tip and washed twice with PBS. The invasion assay was performed using transwells (Millipore, Germany) with an 8- $\mu \mathrm{m}$ pore size polycarbonate membrane filter coated with a layer of $10 \mathrm{mg} / \mathrm{ml}$ matrigel (354248, Corning, USA). Briefly, cells were placed on the upper surface of the transwell insert with (invasion) and without (migration) matrigel.

\section{Cell immunofluorescence assay}

The transfected cells were fixed in $4 \%$ paraformaldehyde for 10 min and washed twice with PBS, blocked with 5\% normal goat serum, and then incubated overnight at $4^{\circ} \mathrm{C}$ with antibody. Thereafter, cells were incubated with fluorescence conjugated secondary antibodies for $1 \mathrm{~h}$ at room temperature. Later, cells were incubated with DAPI for $5 \mathrm{~min}$ in order to stain the nucleus and coverslips were mounted onto the slides. Cells were observed under Olympus IX-71 inverted microscope, and images were taken.

\section{Luciferase target assay}

The putative binding sites of miR-135b-5p in the 3'untranslated region (UTR) of the human $S D C B P$ gene were predicted by combinatorial utilization of two different algorithms, including mirdbV5 (http://mirdb.org/miRDB) and Taregetscan7.1 (http://www.targetscan.org/vert_71) . Direct targeting of the SDCBP 3 '-UTR was determined by cloning of the $3^{\prime}$-UTR seed regions and mutated seed regions into separate pGL3 Luciferase Reporter vectors (Promega, USA). Luciferase and Renilla luciferase were measured using a Dual-Luciferase Reporter Assay System (Promega, USA).

\section{Analysis of tumor growth and metastasis in vivo}

Animal studies were approved by the Institutional Animal Care Committee of West China Hospital, Sichuan University. For tumor growth and metastasis study, NOD/SCID female mice (4-week-old) were randomly divided into two groups (n $=3$ ). The MDA-MB-231 cells were treated with antagomiR$135 \mathrm{~b}-5 \mathrm{p}(200 \mathrm{nmol})$ or antagomiR-NC (200 nmol) for 2 days.

For xenograft tumor model, MDA-MB-231 were inoculated into mammary fat pads of NOD/SCID mice. The tumor volumes were measured weekly throughout the treatment course. Tumor volume was estimated according to the following formula: volume $=$ (longest diameter $\times$ shortest diameter $\left.{ }^{2}\right) / 2$. When tumor burden became 100 $\mathrm{mm}^{3}$, the mice were then treated with a multi-central intratumoral injection of antagomiR-135b-5p (5 nmol) or antagomiR-NC (5 nmol) into appropriate groups once a week for 4 weeks $[17,18]$. 


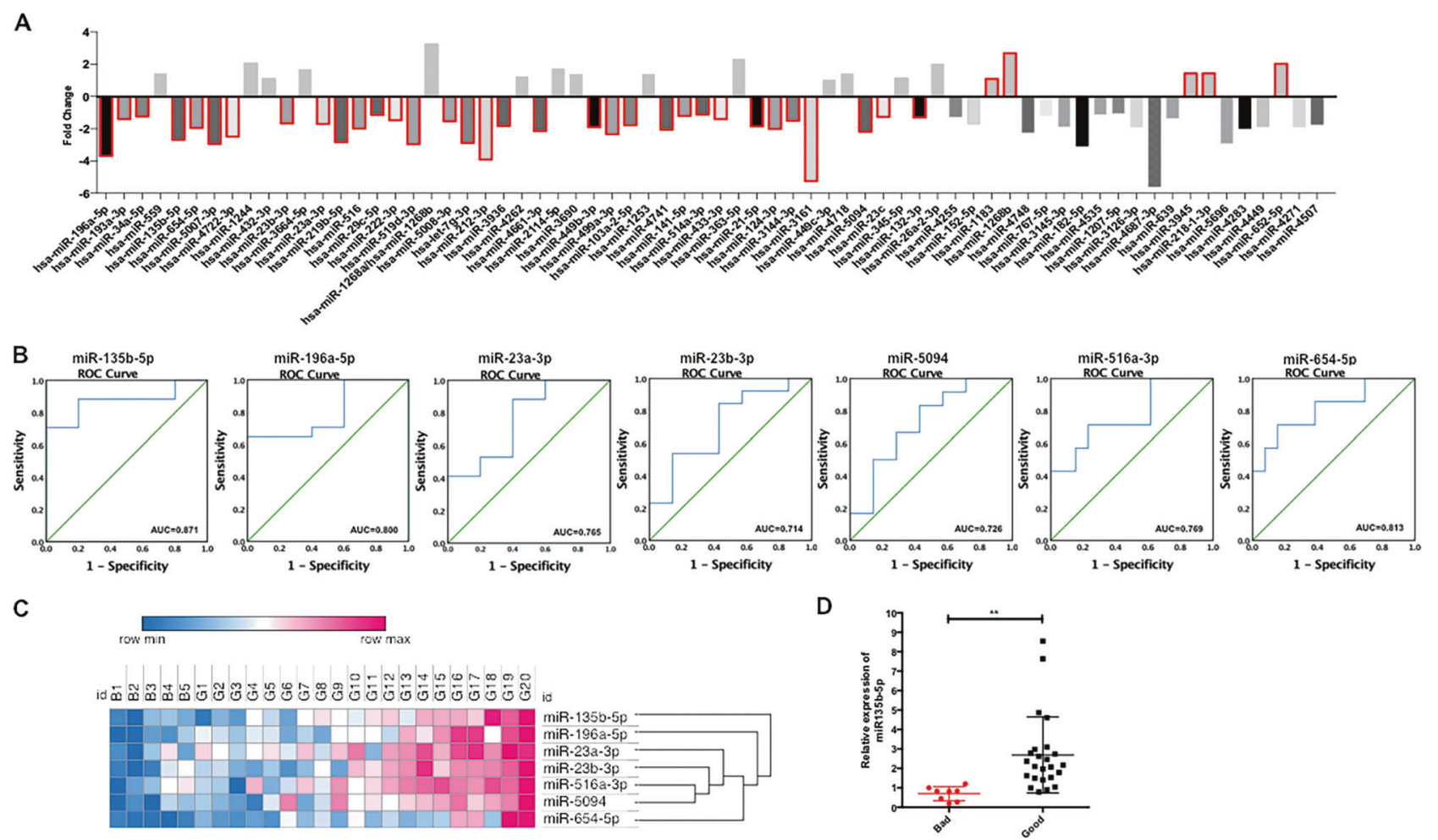

Fig. 1 Screening of miRNAs in early $\mathrm{BC}$ metastasis. a 68 prognostic miRNAs confirmed by qRT-PCR. 38 miRNAs with red line consistent with the previous microarray data. b Receiver operating characteristic curve of the 7 candidate miRNAs with AUC $>0.7$. c Hierarchical

For lung metastasis study, the mice were injected with $10^{7}$ MDA-MB-231 cells into the lateral tail vein. Antagomir$135 \mathrm{~b}-5 \mathrm{p}$ or antagomir-NC was injected into the tail vein of mice per week (diluted in PBS at $80 \mathrm{mg} / \mathrm{kg}$ ) [19, 20].

\section{Statistical analyses}

All the experiments in vitro were performed in triplicate and repeated three times. Statistical significance in cell proliferation, migration and invasion assays as well as luciferase reporter assays was determined by two-tailed Student's $t$-test. The correlation between the expression of miR-136b-5p and clinicopathological features was examined by Pearson's $\chi^{2}$ test. SPSS version 23.0 software (SPSS Inc, IL, USA) was used for statistical analysis. All figures were produced by GraphPad PRISM 6.0. $p$-values of $<0.05$ were considered statistically significant.

\section{Results}

\section{Screening of metastasis-related miRNAs in early BC}

We retrospectively reviewed 242 cases of DCIS-Mi. For all the patients, the clinicopathological and prognostic features cluster analysis showed miR-135b-5p had a significantly different expression pattern in the metastasis samples. $\mathbf{d}$ The expression of miR$135 \mathrm{~b}-5 \mathrm{p}$ was verified by qRT-PCR in different prognosis and consistently low in poor prognosis

were reported as previously published [11]. After a median follow-up of 109 months (6-206 months), among all of the DCIS-Mi metastases, 5 were lung metastases, 2 were liver metastases, and 1 was a bone metastasis.

To screen high-risk miRNAs that lead to metastasis for DCIS-Mi, we reanalyzed the microarray data of Li et al. [16] from a previous study. After data preprocessing, a total of 68 miRNA dysregulation events were screened out as primary candidate molecules for further validation. Compared with the group with good prognosis, 21 miRNAs were upregulated and 47 miRNAs were downregulated in the metastasis group (Supplementary Fig. 1). To further confirm the data from the microarray, 32 DCIS-Mi samples were included for qRT-PCR assay (Supplementary Table 4). There were 38 miRNAs (5 miRNAs upregulated, 33 miRNAs downregulated, Supplementary Table 5) consistent with the microarray data, which were considered potential biomarkers for promoting metastasis for DCIS-Mi (Fig. 1a). The area under the curve (AUC) in the receiver operating characteristic curve (ROC) may have clinical utility as a prospective tool for prediction. We listed 7 candidate miRNAs with AUC $>0.7$, including miR-135b5p, miR-196a-5p, miR-23a-3p, miR-23b-3p, miR-516a-3p, miR-5094, and miR-654-5p (Fig. 1b). Among them, the AUC area of miR-135b-5p was the largest $(\mathrm{AUC}=0.871$ ), 
suggesting a good predictive effect of prognosis for miR135b-5p.

\section{Association of miR-135b-5p with clinicopathological features}

After hierarchical clustering, miR-135b-5p was found to have a significantly different expression pattern in the metastasis samples (Fig. 1c). The miR-135b-5p expression level was consistently downregulated in metastasis samples compared with the nonmetastatic patient samples (Fig. 1d). To analyze the association between miR-135b-5p expression level and clinicopathological characteristics of DCIS$\mathrm{Mi}$, we used the median expression level to dichotomize all the DCIS-Mi cases. Using a high-low cutoff, we found that low miR-135b-5p expression levels were associated with high nuclear grade $(p=0.043)$ and lymph node metastasis $(p=0.031)$ (Table 1).

\section{Suppressing miR-135b-5p promotes cell proliferation, migration, and invasion}

To explore the potential mechanisms of miR-135b-5p, different BC cell lines were used for further study, including MCF-7 (luminal type), SK-BR-3 (HER2 overexpression type), and MDA-MB-231 (basal-like type). With MCF-10A (immortalized normal breast epithelial cells) as a control, the endogenous miR-135b-5p expression level was increased in all BC cell lines in this study (Fig. 2a). To determine the tumor-suppressor properties of miR-135b-5p, MCF-7, SK-BR-3, and MDA-MB-231 cells were transfected with a miR-135b-5p inhibitor for functional analysis, and the inhibition efficiency was confirmed by qRT-PCR (Fig. 2b).

Cell motility was first evaluated by a wound-healing assay in cells with or without miR-135b-5p silencing. As shown in Fig. 2c, inhibiting miR-135b-5p markedly increased the migration rates of MDA-MB-231 cells. The difference became significant $24 \mathrm{~h}$ after wounding and became more evident over time $(p<0.001)$. The difference in migration capability was further confirmed by transwell assay. Similarly, cells with miR-135b-5p silencing migrated more efficiently through the micropore wells than the cells treated with control. The increased migration rate ranged from two to threefold in a cell type-dependent manner $(p<$ 0.001 , Fig. 2d). Further invasion assays showed that suppressed miR-135b-5p promoted both migration and invasion in BC cells (Fig. 2e).

The CCK-8 and colony formation assays demonstrated that suppressing miR-135b-5p with an inhibitor led to a significant increase in BC cell proliferation (Fig. 2f, g). However, apoptosis assays showed that miR-135b-5p did not affect apoptosis (Fig. 2h).
Table 1 Association of miR-135b-5p expression with clinicopathological features

\begin{tabular}{|c|c|c|c|c|}
\hline & \multicolumn{2}{|c|}{ miR-135b-5p } & \multirow[t]{2}{*}{$\chi$} & \multirow[t]{2}{*}{$p$-value } \\
\hline & Low $(\%)$ & High $(\%)$ & & \\
\hline Age (yrs) & & & 1.348 & 0.246 \\
\hline$<50$ & $10(71.4)$ & $7(50.0)$ & & \\
\hline$\geq 50$ & $4(28.6)$ & $7(50.0)$ & & \\
\hline Tumor size $(\mathrm{cm})$ & & & 2.800 & 0.094 \\
\hline $\mathrm{T} \leq 5$ & $8(57.1)$ & $12(85.7)$ & & \\
\hline $\mathrm{T}>5$ & $6(42.9)$ & $2(14.3)$ & & \\
\hline Nuclear grade & & & 4.094 & $0.043 *$ \\
\hline Low-mid & $2(14.3)$ & $7(50.0)$ & & \\
\hline High & $12(85.7)$ & $7(50.0)$ & & \\
\hline Necrosis & & & 2.489 & 0.115 \\
\hline Yes & $3(21.4)$ & $7(50.0)$ & & \\
\hline No & $11(78.6)$ & $7(50.0)$ & & \\
\hline Lymph node status & & & 4.667 & $0.031 *$ \\
\hline Negative & $10(71.4)$ & $14(100)$ & & \\
\hline Positive & $4(28.6)$ & 0 & & \\
\hline ER status & & & 2.286 & 0.131 \\
\hline Negative & $9(64.3)$ & $5(35.7)$ & & \\
\hline Positive & $5(35.7)$ & $9(64.3)$ & & \\
\hline PR status & & & 0.164 & 0.686 \\
\hline Negative & $10(71.4)$ & $9(64.3)$ & & \\
\hline Positive & $4(28.6)$ & $5(35.7)$ & & \\
\hline HER2 status & & & 0.622 & 0.430 \\
\hline Negative & $8(57.1)$ & $10(71.4)$ & & \\
\hline Positive & $6(42.9)$ & $4(28.6)$ & & \\
\hline Ki-67 index & & & 0.583 & 0.445 \\
\hline Ki-67 $\leq 14$ & $5(35.7)$ & $7(50.0)$ & & \\
\hline Ki-67 > 14 & $9(64.3)$ & $7(50.0)$ & & \\
\hline
\end{tabular}

HER2 status were confirmed by immunohistochemistry (IHC) and fluorescence in situ hybridization (FISH)

$D C I S-M i$ ductal carcinoma in situ with microinvasion, ER estrogen receptor, $P R$ progesterone receptor, HER 2 human epidermal growth factor receptor 2

*Statistically significant

Taken together, these data indicate that miR-135b-5p has an important role in $\mathrm{BC}$ progression.

\section{Inhibition of miR-135b-5p promotes EMT}

The epithelial-mesenchymal transition (EMT) is a critical event in the cancer metastasis process. Cells undergoing EMT exhibit elevated cell motility with high-grade malignancy. Therefore, we further examined the impact of miR135b-5p on EMT. miR-135b-5p suppression was paralleled with the repressed expression of epithelial marker CDH1 (E-cadherin) and the upregulated expression of mesenchymal markers Vim and Snail (Fig. 3a). In addition, the miR- 


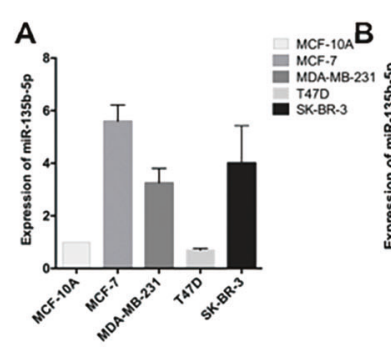

C
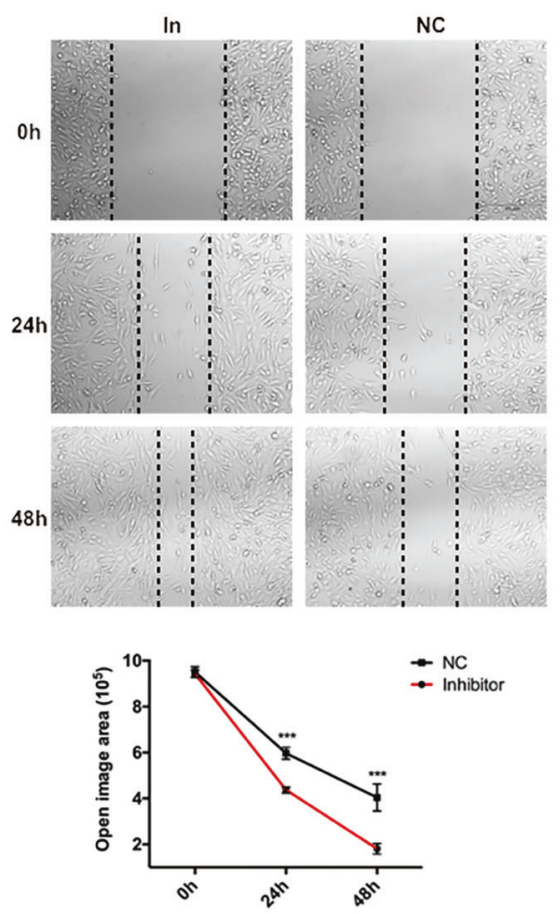
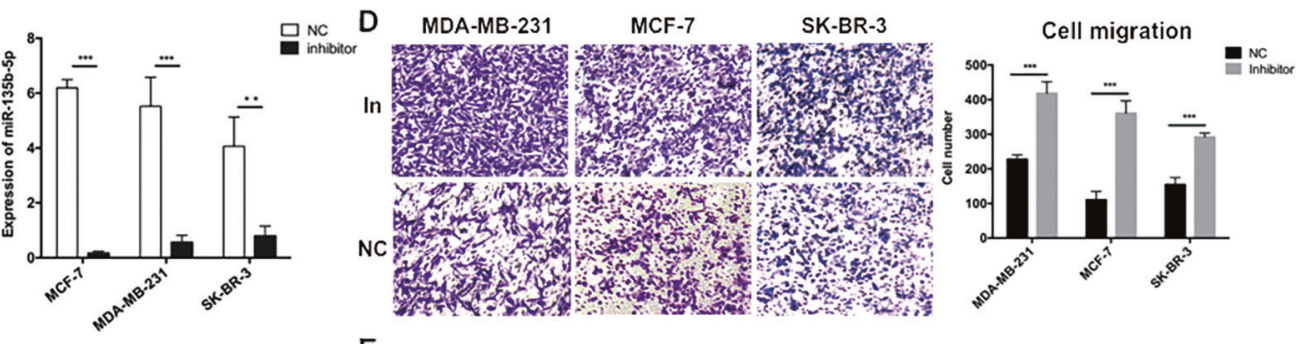

E MDA-MB-231

MCF-7

SK-BR-3
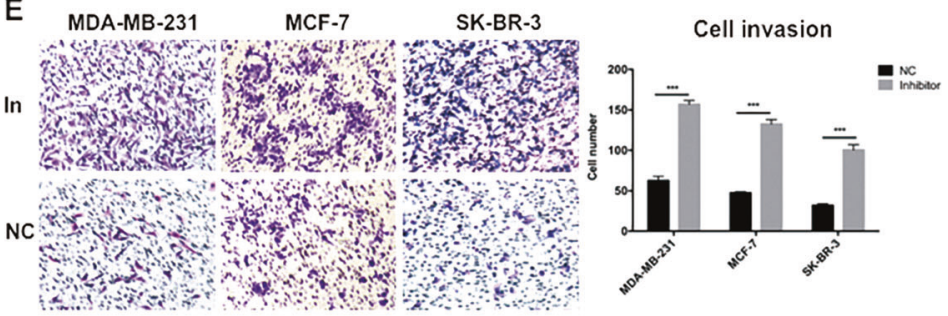

F
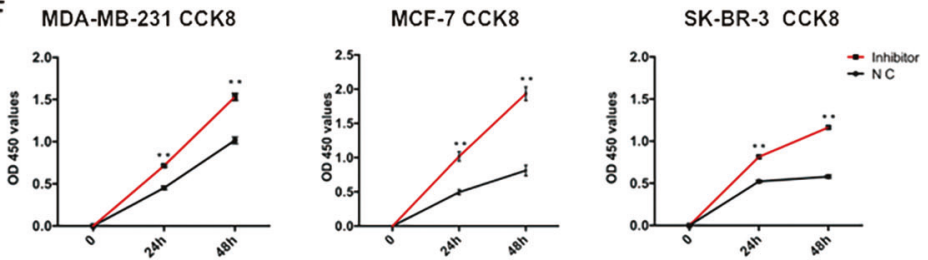

G
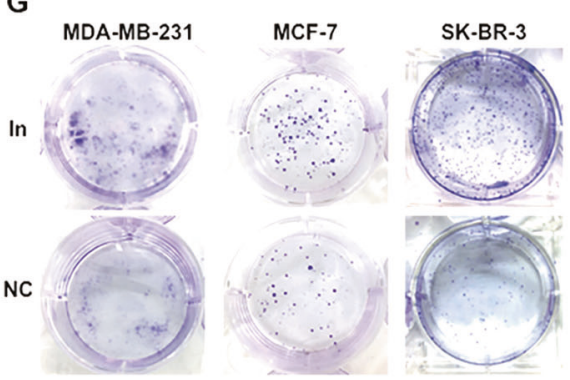

$\mathrm{H}$

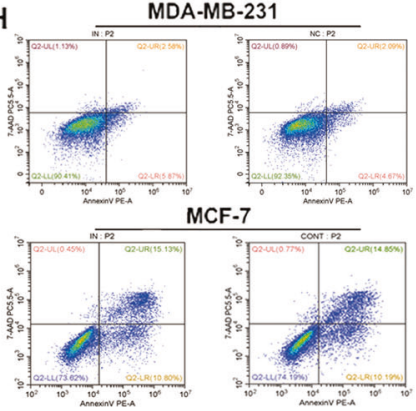

determined after transduction with the miR-135b-5p inhibitor (In) or NC. f CCK-8 assay for BC cells proliferative activity in different treatment groups and different times after transfection. $\mathrm{g}$ Colony formation assay for $\mathrm{BC}$ cells proliferative activity in different groups. h Apoptosis assays showed that miR-135b-5p did not affect cell apoptosis. These results were representative of at least three independent experiments. Error bars, mean \pm S.E.M. $* p<0.05, * * p<0.01$

predicted by the two commonly cited miRNA-mRNA bioinformatics tools mirdbV5 and Taregetscan7.1 (Supplementary Fig. 2A). Based on the frequencies of miR-135b-5p sites in their 3'-UTRs, 104 target mRNAs were predicted by at least two databases (Fig. 4a). Guided by algorithms and the literature, we evaluated 12 putative miR-135b-5p targets: ANGPTL2, BACH1, BCAT1, KLF4, LATS2, MRAS, PHLDB2, PIK3R2, PPM1E, RALBP1, SDCBP, and SIAH1 that have previously been shown to be involved in tumorigenesis and progression. We investigated the mRNAlevel effects of miR-135b-5p inhibition on these genes by transfecting MDA-MB-231 with miR-135b-5p inhibitor, which significantly increased the LATS2, RALBP1and SDCBP mRNA levels (Supplementary Fig. 2B). SDCBP

tumor progression, the potential mRNA targets were 


\section{A}

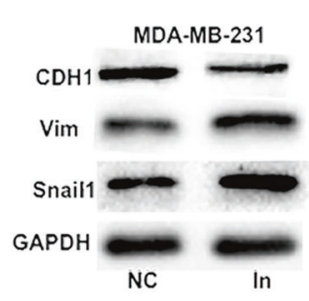

C
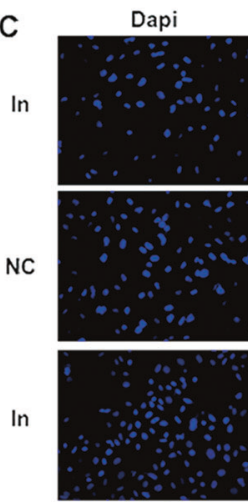

NC
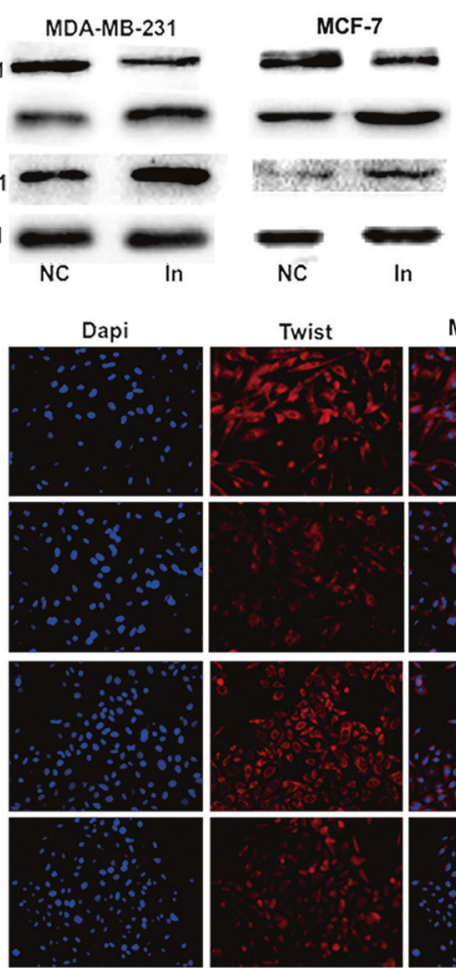

In

Twist

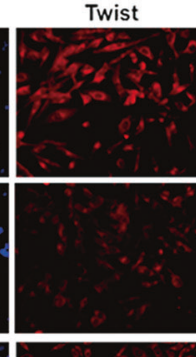

Merged view

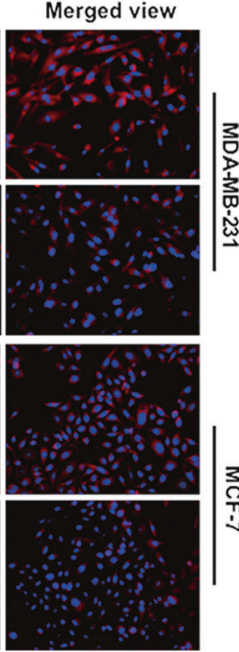

Fig. 3 Inhibition of miR-135b-5p promote the EMT of BC cells. a, b Western blot and qRT-PCR determined the protein and mRNA level of CDH1, Vim and Snail1 in BC cells suppressing miR-135b-5p inhibitor or NC. GAPDH was used as an internal control. c The Immunostaining analysis of Twist expression $(\times 200$ magnification). d Image showing the rearrangement of actin cytoskeleton of

was the only protein that inversely correlated with miR135b-5p expression (Fig. 4b). In addition, the regulation of SDCBP by miR-135b-5p was a common event, which was also repeated in the MCF-7 and SK-BR-3 cell lines. These findings suggested that the increased SDCBP expression was most likely the direct result of decreased miR-135b-5p expression in different BC cells. SDCBP has been reported to affect cytoskeletal-membrane organization and localization to membrane-associated adherens junctions and focal adhesions. Thus, SDCBP was selected for further analysis in this study.

Based on the bioinformatics analysis, there was one single potential miR-135b-5p binding site at the SDCBP $3^{\prime}-\mathrm{UTR}$. The complementarity of the SDCBP $3^{\prime}$-UTR with the seed sequence of miR-135b-5p is shown in Fig. 4c. To study the direct relation between SDCBP and miR-135b-5p, SDCBP mRNA 3'-UTR fragments (either wild type or mutant) were constructed and cotransfected with the miR-135b-5p plasmid into $293 \mathrm{~T}$ cells. Remarkably, miR-135b-5p repressed the luciferase activity in the wild-type $3^{\prime}$-UTR of SDCBP (Fig. 4c). This phenomenon was not detected in the mutantbinding sites, suggesting that the regulation of SDCBP by miR-135b-5p is sequence specific. Taken together, these

\section{B}

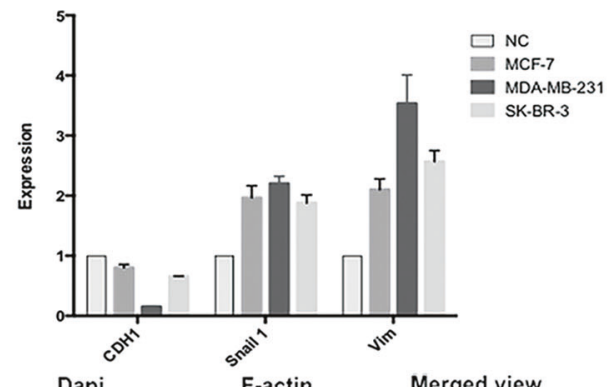

D

In
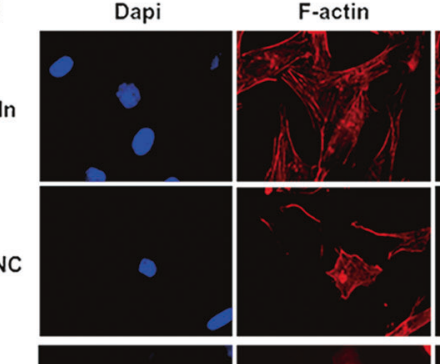

Merged view

In

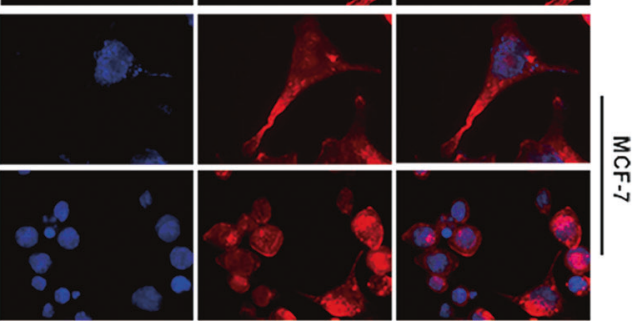

MDA-MB-231 and MCF-7 after transduction with the miR-135b-5p inhibitor or NC ( $\times 600$ magnification). Stress fibers and actin filaments were visualized by Rhodamine-phalloidin staining (red). Nuclei were stained with DAPI (blue). These results were representative of at least three independent experiments. Error bars mean \pm S.E.M.

results suggest that miR-135b-5p downregulates SDCBP expression by directly targeting its $3^{\prime}$-UTR.

\section{Expression of SDCBP in BC samples}

We globally analyzed the SDCBP expression profiles of DCIS, DCIS-Mi and IDC tissues using immunohistochemistry (IHC). Progression of BC was accompanied by increased expression of SDCBP. The SDCBP expression level of tumor sections was upregulated in metastasis patients compared with nonmetastasis patients (Fig. 4d). The validation experiments were performed using qRTPCR, and the SDCBP expression pattern was confirmed (Fig. 4e).

\section{Knockdown of SDCBP reverses the effect of miR- $135 b-5 p$ function in vitro}

To assess the functional contributions of the target gene to the aggressive phenotypes of $\mathrm{BC}$ cells, we further examined the contribution of SDCBP in miR-135b-5p-dependent EMT by functional rescue experiments. Notably, the CCK8 , colony formation and transwell assays showed that 

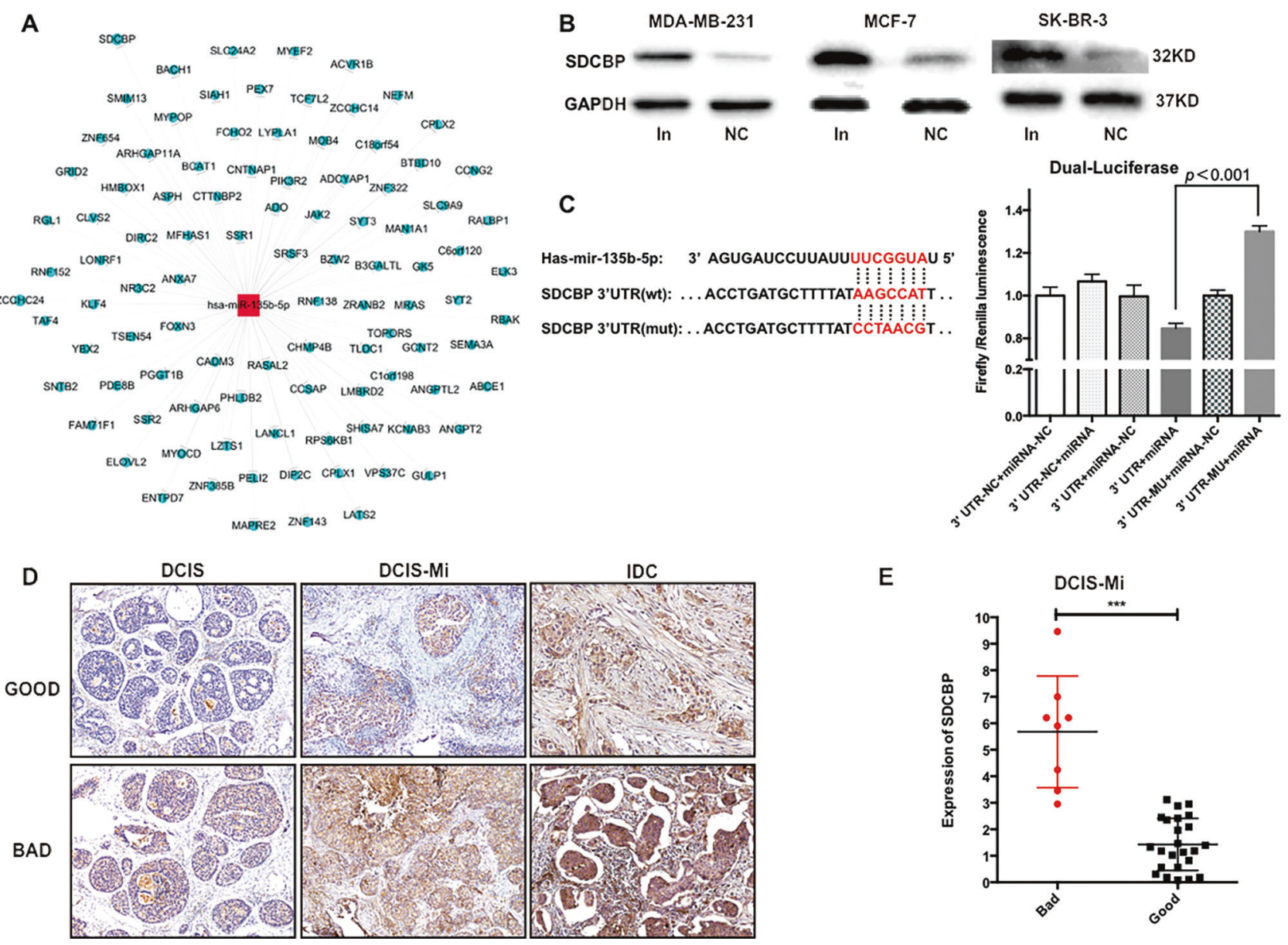

E

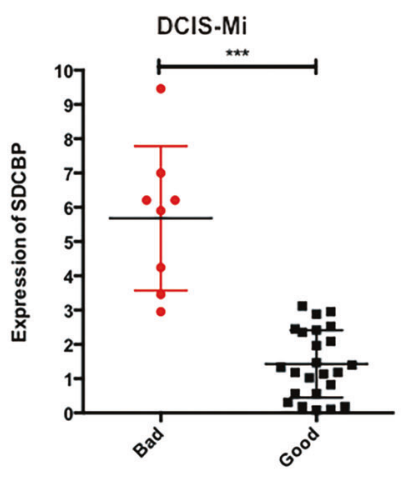

Fig. 4 SDCBP was the direct target of miR-135b-5p. a 104 putative target genes of miR-135b-5p. b Western blot analysis of SDCBP at 48 h post-transfection with either MiR-135b-5p inhibitors/NC. GAPDH was used as loading controls. $\mathbf{c}$ Sequences of the miR-135b-5p binding sites in wild type and mutant (red) SDCBP 3 '-UTR. The luciferase activities of wild type or mutant 3'-UTR SDCBP luciferase reporter

similar to the effects of miR-135b-5p inhibition, rescue with the SDCBP-plasmid was associated with a significantly increased level of cell proliferation and enhanced motility (Fig. 5a-c). Next, we examined whether SDCBP knockdown could reverse the effects. The in vitro results revealed that SDCBP-siRNA could partially reverse the effects of miR-135b-5p inhibition on proliferation and motility (Fig. 5a-c) in different cell lines. In addition, the ability of the miR-135b-5p inhibitor to regulate EMT, as determined by CDH1 suppression, Vim and Snail expression, was largely abrogated by the downregulation of SDCBP (Fig. 5d). The above evidence indicates that SDCBP is indeed a functional target of miR-135b-5p in $\mathrm{BC}$ cells.

\section{Suppressing miR-135b-5p promotes tumor growth and lung metastasis in vivo}

To confirm the tumor suppression role of miR-135b-5p in vivo, we used a xenograft tumor model for functional study were measured after transfection with NC or miR-135b-5p. d SDCBP immunoreactivity was detected in DCIS, DCIS-Mi, and IDC tumor with or without metastases. e The expression of SDCBP was verified by qRT-PCR in DCIS-Mi with different prognosis. Error bars, mean \pm S.E.M. $* p<0.05, * * p<0.01$

(Fig. 6a). Pretransfected MDA-MB-231 cells were injected into mammary fat pads of NOD/SCID mice. The difference in tumor size between the two groups became statistically significant after 4 weeks injection and became increasingly evident over time (Fig. 6b). As expected, the tumor size was significantly increased after suppressing miR-135b-5p expression in cells (Fig. 6c). H\&E staining showed that, compared with the control group, the tumors in the inhibition group were more malignant, showing higher nuclear grade and more pathological mitosis (Fig. 6d). The downregulation of miR-135b-5p and overexpression of SDCBP were confirmed in the dissected xenograft, demonstrating that the silencing effect of antagomiR-135b-5p was long-lasting (Fig. 6e). Consistent with the previous results, the inhibition of miR-135b-5p promoted tumor cell growth and proliferation and showed overexpressed proliferation-related genes (MKI67, STK5 and Survivin, $p<0.01$ ) in the mammary tumors (Fig. 6f). Moreover, lung metastasis was observed in $3 / 3$ mice 6 weeks after tumor cell injection (Fig. 6g). No 


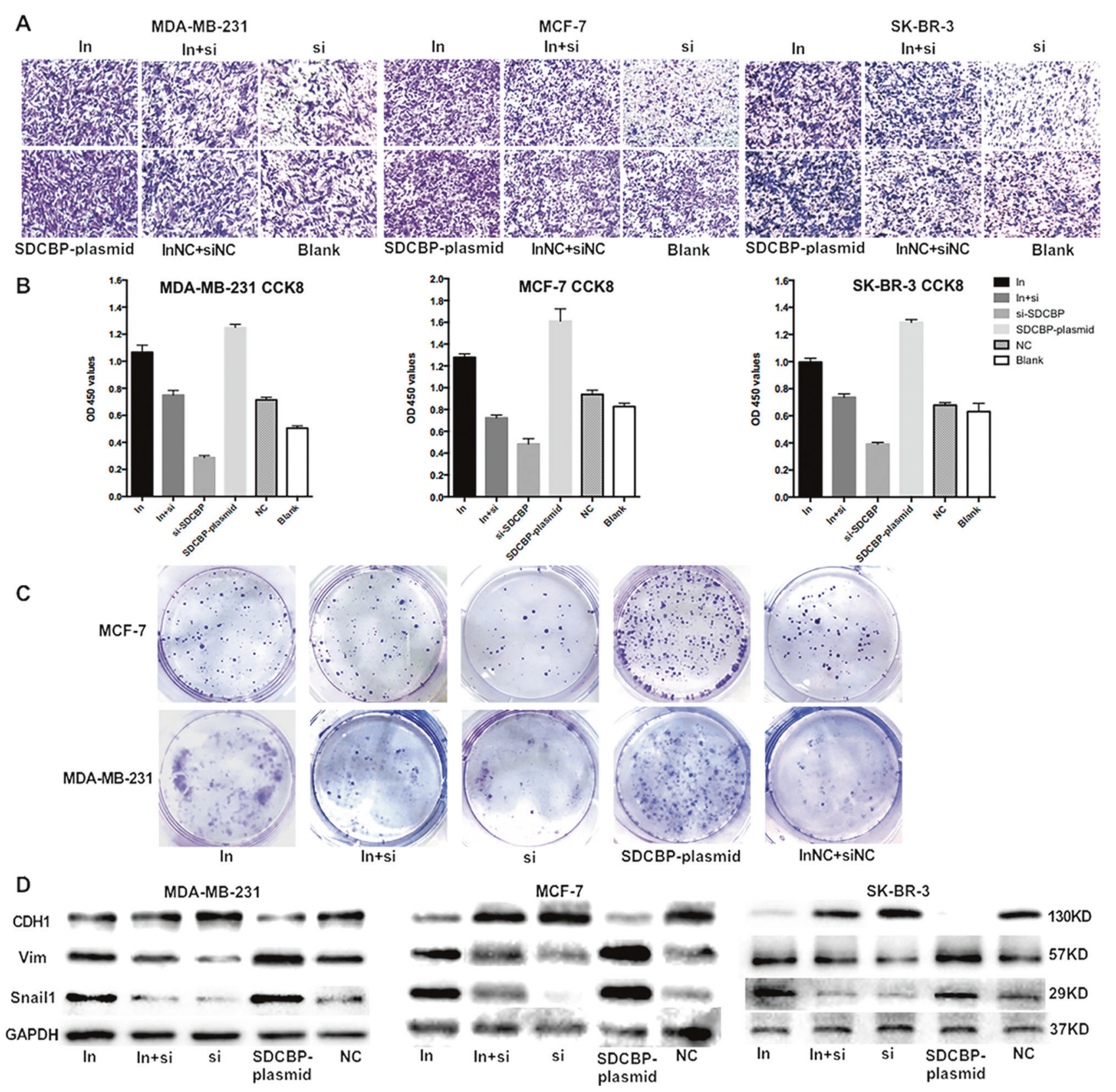

Fig. 5 Knockdown of SDCBP partially rescued cell migration and proliferation by downregulation of miR-135b-5p in BC. a-c Transwell migration, CCK-8 and colony formation assays were performed in $\mathrm{BC}$ cells after transfection with miR-135b-5p inhibitor, si-SDCBP,
SDCBP-plasmid, and NC. d The expressions of EMT markers were determined by western blot in BC cells. GAPDH was used as an internal control. These results were representative of at least three independent experiments. Error bars mean \pm S.E.M. metastasis was found in other organs. However, no lung metastasis was found in the control group. In addition, antiagomiR-135b-5p increased Vim expression and decreased CDH1 expression $(p<0.01$, Fig. $6 \mathrm{f})$.

Next, we examined the effect of miR-135b-5p on metastasis by metastasis model. We injected MDA-MB-231 cells pretreated with antagomiR-135b-5p. AntagomiR$135 \mathrm{~b}-5 \mathrm{p}$ or NC was injected intravenously to suppress miR-135b-5p systemically (Fig. 7a). Four weeks after systemic injection, mice were harvested for analysis. Histology slides for the lungs confirmed the metastasis foci. The tumor nodules throughout the pulmonary region were markedly increased in the inhibition group compared with the NC group (Fig. 7b). No metastasis was found in other organs. In addition, qRT-PCR and western blot analysis revealed that SDCBP expression at the mRNA and protein levels increased in the miR-135b-5p antagomir-treatment group relative to the controls (Fig. 7c). Consistent with the results of the previous experiments, miR-135b-5p suppression markedly enhanced the ability of SDCBP to promote the 


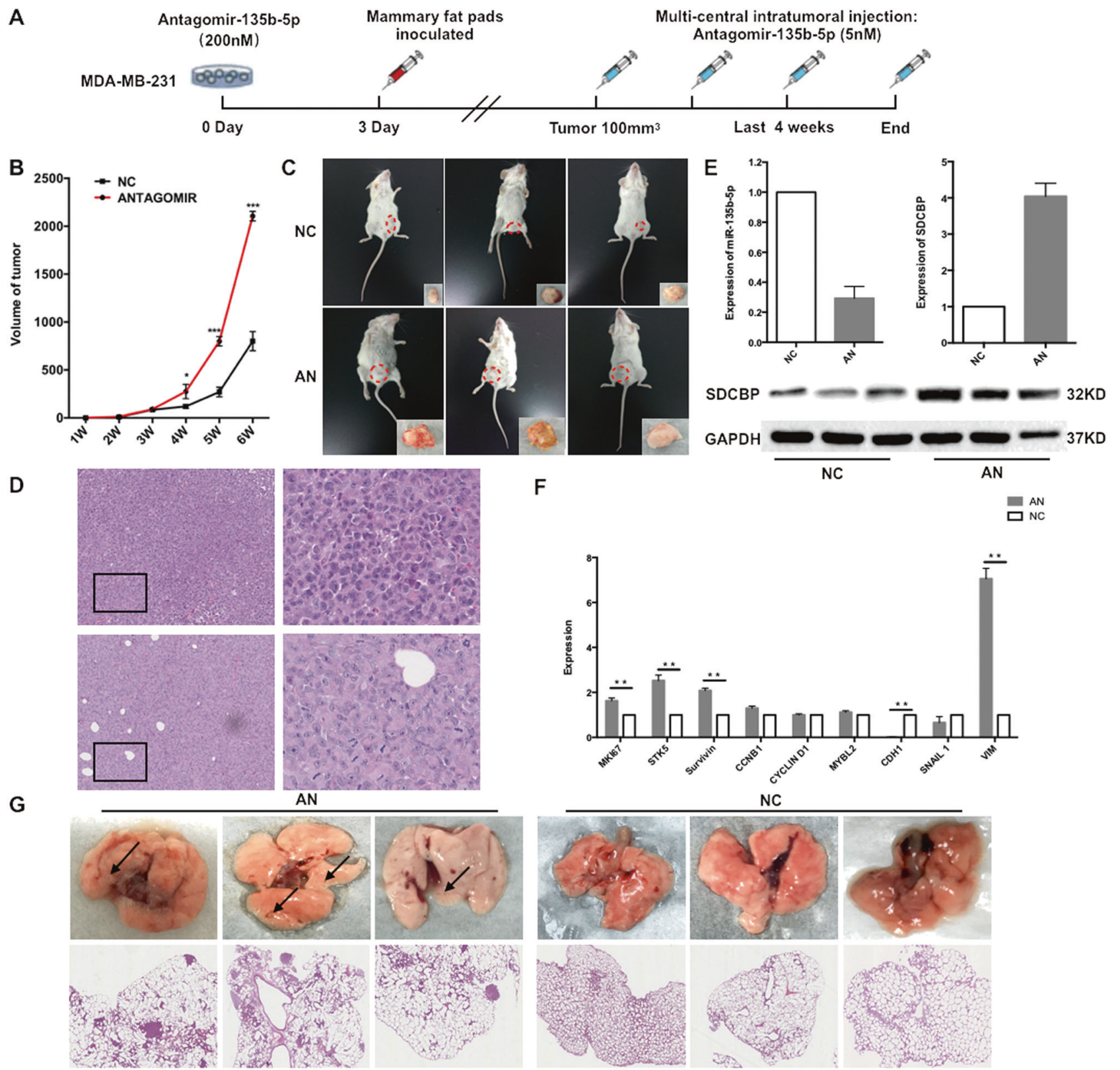

Fig. 6 Antagomir-135b-5p can be directly delivered to tumor cells in vivo and promote cell proliferation, motility, and invasiveness. a Schematic representation of the antagomir administration schedule for xenograft tumor model. b, c Volume of primary mice mammary tumors formed by MDA-MB-231 cells infected with Antagomir-135b$5 p$ or NC. d H\&E stain of the mammary tumors (left: $\times 40$ magnification, right: $\times 400$ magnification). e miR-135b-5p and SDCBP mRNA

metastasis of BC cells to the lung. This result was accompanied by high MKI67, STK5, Survivin, and Cyclin D1 expression levels $(p<0.01$, Fig. $7 d)$.

\section{Discussion}

DCIS-Mi is an uncommon pathological entity accounting for $1 \%$ of all $\mathrm{BC}$ cases [1]. The biomarkers associated with

and protein expression in the mammary tumors. GAPDH was used as an internal control. $\mathbf{f}$ The expressions of proliferation-related genes and EMT markers were determined by qRT-PCR in the mammary tumors. g Bright field imaging and H\&E stain of the lungs and number of visible lung metastases ( $\times 200$ magnification). Arrows indicate lung metastases. ( $n=3$ mice in each group). Error bars, mean \pm S.E.M. ${ }^{*} p<0.05, * * p<0.01$

prognosis in DCIS-Mi and the mechanism of recurrence and metastasis are not clear. The development of a better algorithm to identify a "metastasis" subgroup may increase the overall survival for DCIS-Mi patients by individualized treatment and early prevention.

DCIS-Mi is particularly important because it occurs at the very beginning stage of IDC. In routine clinical practice, the entire specimen should be examined, and all tissues likely involved by DCIS should be complete submission. 

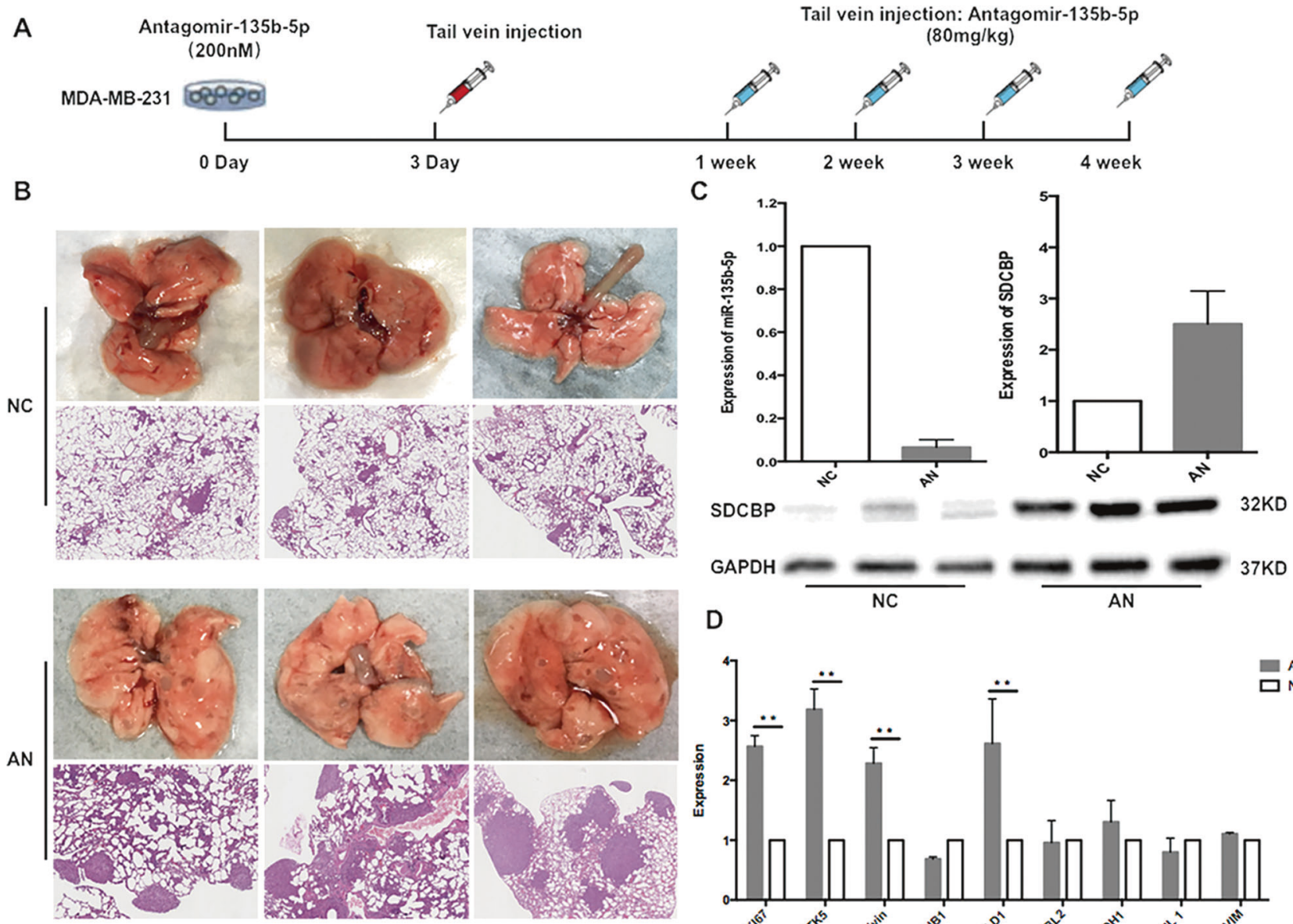

D

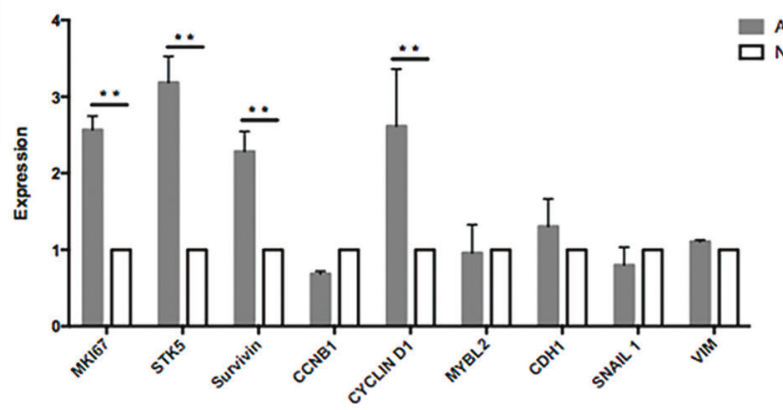

Fig. 7 Antagomir-135b-5p treatment affected late stages of the metastatic process. a Schematic representation of the antagomir administration schedule for mice with tail vein injection of MDA-MB-231 cells. b Bright field imaging and $\mathrm{H} \& \mathrm{E}$ stain of the lungs and number of visible lung metastases in NC- or antagomir-135b-5p-treated mice

Pathologists will be required complete microscopic examination to find microinvasion in slides. For this reason, DCIS-Mi samples are difficult to collect, and the molecular markers with prognostic value are especially important for DCIS-Mi patients.

In $\mathrm{BC}$, some dysregulated miRNAs promote cancer invasion and metastasis. MiRNAs are more stable than mRNA, protein and other molecules. It has been reported that the quality of miRNA in tissues remains eligible over 10 years [12-15]. This property makes miRNAs useful for long-term follow-up research, especially for tumors with a relatively good prognosis, such as DCIS-Mi or early-stage BC. In the current study, we compared the miRNA profiles of DCIS-Mi with or without metastasis by microarray and qRT-PCR. A total of seven miRNAs (miR-135b-5p, miR196a-5p, and miR-23a- 3p, miR-23b-3p, miR-516a-3p, miR-5094, and miR-654-5p), which had key roles in malignant progression were found to be differentially expressed across the two groups. The AUC of miR-135b-5p
( $\times 200$ magnification). $\mathbf{c}$ The expression of miR-135b-5p and SDCBP in lungs of mice with subsequent treatment with $\mathrm{NC}$ or antagomir135b-5p. GAPDH was used as an internal control. d qRT-PCR of proliferation-related genes and EMT markers. $(n=3$ mice in each group). Error bars, mean \pm S.E.M. $* p<0.05, * * p<0.01$

was the largest $(\mathrm{AUC}=0.871)$, representing a good prediction. Correlation analysis revealed that decreased miR135b-5p expression was associated with high nuclear grade and lymph node metastasis. Therefore, miR-135b-5p was chosen as the focus of this study.

MiR-135b-5p is a new miRNA, and its molecular mechanism is still unknown. Chen et al pointed out that miR-135b-5p was downregulated in both human gastric cancer tissues and cells. As one of the miR-135b-5p target genes, PPM1E upregulation is important for cell proliferation, possibly via the regulation of AMPK-mTOR signaling [21], which is consistent with the research results of $\mathrm{Li}$ et al. [22]. However, we did not find a correlation between PPM1E and miR-135b-5p in ER-negative BC cell lines (MDA-MB-231 and SK-BR-3). The regulation of PPM1E is conditional and limited by the cell type and microenvironment. However, evidence for an oncogenic role of miR-135b-5p has also begun to accumulate. For instance, Han et al. [23] observed the upregulation of miR-135b-5p in 
pancreatic cancer. In addition, the inhibition of miR-135b$5 p$ has been associated with a decrease in the proportion of stem cells in pancreatic cancer [24]. These results seem controversial. Despite detailed mechanisms remaining unknown, one potential explanation is that the same miRNA may have multiple targets in different cells and may interact with the microenvironment to select which target to regulate in a specific situation. For example, miR-205 has a double-edged sword effect on EMT under hypoxia or hyperoxia [25-27]. Another alternative explanation is that miRNA fulfills its task via binding to the 3'-UTR region of target mRNA. The expression of oncogenes can be regulated by numerous mechanisms, such as gene deletion or epigenetic promoter methylation and histone modification. Therefore, if the oncogenic targets of miR-135b-5p, such as NR3C2 [28], are suppressed by the abovementioned mechanisms, no targeting mRNA would be available for miR-135b-5p to bind, and the genes would not become carcinogenic. Instead, miR-135b-5p may selectively regulate its tumor-suppressed target, such as PPM1E, and thus promote EMT in malignancies.

We detected miR-135b-5p overexpression in 3 different types of BC cells (MDA-MB-231, MCF-7, SK-BR-3), suggesting that miR-135b-5p has an important role in the development of tumors. However, miR-135b-5p was downregulated in metastatic BC samples. In addition, the inhibition of miR-135b-5p in BC cells could increase migration and invasion. These findings suggest that the high expression of miR-135b-5p in the early stage can promote the formation of tumor, while the downregulation of miR$135 \mathrm{~b}-5 \mathrm{p}$ can promote the invasion and metastasis of tumor cells. The pleiotropic role of miRNAs in different stages has been elucidated [29-31].

We used luminal (MCF-7), basal-like (MDA-MB-231) and HER2 overexpressing (SK-BR-3) cell lines to explore the role of miR-135b-5p in different molecular subtypes of BC. These cells not only have molecular differences but also have different migration abilities. Here, we identified that SDCBP is a novel target of miR-135b-5p and that the binding site of miR-135b-5p at the $3^{\prime}$-UTR of SDCBP is conserved. The present data clearly indicates that the inhibition of miR-135b-5p and the subsequent upregulation of SDCBP can induce EMT, proliferation, migration and invasion of different BC cells. Conversely, exogenous expression of SDCBP can reverse the biological effect of miR-135b-5p. Moreover, we observed the effects of miR$135 b-5 p$ inhibition in a xenograft tumor model. It is noteworthy that, consistent with the in vitro outcomes, inhibiting miR-135b-5p in vivo increased tumor growth and induced metastasis. However, we found that the EMT markers did not change in the tumor metastases experiment. During the development of cancer, the ability of cells to undergo EMT can lead tumor cells to leave the primary tumor. However, when circulating tumor cells colonize the secondary sites to invade surrounding tissues, EMT is no longer necessary.

Taken together, we have isolated miR-135b-5p as having essential prognostic value for DCIS-Mi patients, and the miR-135b-5p/SDCBP axis has been identified as a novel mechanism for DCIS-Mi metastasis. Thus, our clinical patient cohort and functional data suggest that miR-135b$5 \mathrm{p} / \mathrm{SDCBP}$ is a crucial determinant of BC metastasis at a very early stage. Our results may shed light on the importance of miR-135b-5p molecular diagnosis and prognosis, as well as the early prevention of $\mathrm{BC}$ for metastasis.

Funding This study was supported by 1.3 .5 project for disciplines of excellence, West China Hospital, Sichuan University (ZYGD18012) and the Research Supporting Project from Science and Technology Department of Sichuan Province (nos. 2017SZ0005, 2019YFS0324, and 2018JY0277), and Research Project from Health Commission of Sichuan Province (16PJ335).

\section{Compliance with ethical standards}

Conflict of interest The authors declare that they have no conflict of interest.

Publisher's note: Springer Nature remains neutral with regard to jurisdictional claims in published maps and institutional affiliations.

\section{References}

1. Bianchi S, Vezzosi V. Microinvaive carcinoma of the breast. Pathol Oncol Res. 2008;14:105-11.

2. Wang L, Zhang W, Lyu S, Liu X, Zhang T, Liu S, et al. Clinicopathologic characteristics and molecular subtypes of microinvasive carcinoma of the breast. Tumour Biol. 2015;36:2241-8.

3. Fan L, Zheng Y, Yu KD, Liu GY, Wu J, Lu JS, et al. Breast cancer in a transitional society over 18 years: trends and present status in Shanghai, China. Breast Cancer Res Treat. 2009;117:409-16.

4. Edge SB, Compton CC. The American Joint Committee on Cancer: the 7th edition of the AJCC cancer staging manual and the future of TNM. Ann Surg Oncol. 2010;17:1471-4.

5. Parikh RR, Haffty BG, Lannin D, Moran MS. Ductal carcinoma in situ with microinvasion: prognostic implications, long-term outcomes, and role of axillary evaluation. Int J Radiat Oncol Biol Phys. 2012;82:7-13.

6. Margalit DN, Sreedhara M, Chen YH, Catalano PJ, Nguyen PL, Golshan M, et al. Microinvasive breast cancer: ER, PR, and HER-2/neu status and clinical outcomes after breast-conserving therapy or mastectomy. Ann Surg Oncol. 2013;20:811-8.

7. Fang Y, Wu J, Wang W, Fei X, Zong Y, Chen X, et al. Biologic behavior and long-term outcomes of breast ductal carcinoma in situ with microinvasion. Oncotarget. 2016;7:64182-90.

8. Matsen CB, Hirsch A, Eaton A, Stempel M, Heerdt A, Van Zee $\mathrm{KJ}$, et al. Extent of microinvasion in ductal carcinoma in situ is not associated with sentinel lymph node metastases. Ann Surg Oncol. 2014;21:3330-5.

9. Thomas A, Weigel RJ, Lynch CF, Spanheimer PM, Breitbach EK, Schroeder MC. Incidence, characteristics, and management of recently diagnosed, microscopically invasive breast cancer by 
receptor status: Iowa SEER 2000 to 2013. Am J Surg. 2017;214:323-8.

10. Wang W, Zhu W, Du F, Luo Y, Xu B. The demographic features, clinicopathological characteristics and cancer-specific outcomes for patients with microinvasive breast cancer: a SEER database analysis. Sci Rep. 2017;7:42045.

11. Tianjie P, Xiaorong Z, Ling D, Shi L, Yan Q, Yueping L, et al. Long term prognosis of ductal carcinoma in situ with microinvasion: a retrospective cohort study. Int J Clin Exp Pathol 2018. 2018;11:2665-74.

12. Siebolts U, Varnholt H, Drebber U, Dienes HP, Wickenhauser $\mathrm{C}$, Odenthal M. Tissues from routine pathology archives are suitable for microRNA analyses by quantitative PCR. J Clin Pathol. 2009;62:84-8.

13. Lawrie CH, Soneji S, Marafioti T, Cooper CD, Palazzo S, Paterson JC, et al. MicroRNA expression distinguishes between germinal center B cell-like and activated B cell-like subtypes of diffuse large B cell lymphoma. Int J Cancer. 2007;121:1156-61.

14. Peiro-Chova L, Pena-Chilet M, Lopez-Guerrero JA, GarciaGimenez JL, Alonso-Yuste E, Burgues O, et al. High stability of microRNAs in tissue samples of compromised quality. Virchows Arch. 2013;463:765-74.

15. Xi Y, Nakajima G, Gavin E, Morris CG, Kudo K, Hayashi $\mathrm{K}$, et al. Systematic analysis of microRNA expression of RNA extracted from fresh frozen and formalin-fixed paraffin-embedded samples. Rna. 2007;13:1668-74.

16. Shi L, Tianjie P, Lin X, Hongwei G, Li L, Feng Y, et al. Screening of recurrence related MiRNA in DCIS and functional study of MiRNA-654-5p. J Breast Cancer. 2019;22:e4.

17. Wang K, Liu CY, Zhou LY, Wang JX, Wang M, Zhao B, et al. APF IncRNA regulates autophagy and myocardial infarction by targeting miR-188-3p. Nat Commun. 2015;6:6779.

18. Ma L, Reinhardt F, Pan E, Soutschek J, Bhat B, Marcusson EG, et al. Therapeutic silencing of miR-10b inhibits metastasis in a mouse mammary tumor model. Nat Biotechnol. 2010;28:341-7.

19. Li H, Xie H, Liu W, Hu R, Huang B, Tan YF, et al. A novel microRNA targeting HDAC5 regulates osteoblast differentiation in mice and contributes to primary osteoporosis in humans. J Clin Invest. 2009;119:3666-77.

20. Thum T, Gross C, Fiedler J, Fischer T, Kissler S, Bussen M, et al. MicroRNA-21 contributes to myocardial disease by stimulating MAP kinase signalling in fibroblasts. Nature. 2008;456:980-4.
21. Chen MB, Liu YY, Cheng LB, Lu JW, Zeng P, Lu PH. AMPKalpha phosphatase Ppm1E upregulation in human gastric cancer is required for cell proliferation. Oncotarget. 2017;8:31288-96.

22. Li ZW, Zhu YR, Zhou XZ, Zhuo BB, Wang XD. microRNA135b expression silences Ppmle to provoke AMPK activation and inhibit osteoblastoma cell proliferation. Oncotarget. 2017;8:26424-33.

23. Han X, Saiyin H, Zhao J, Fang Y, Rong Y, Shi C, et al. Overexpression of miR-135b-5p promotes unfavorable clinical characteristics and poor prognosis via the repression of SFRP4 in pancreatic cancer. Oncotarget. 2017;8:62195-207.

24. Sanchez-Diaz PC, Hsiao TH, Chang JC, Yue D, Tan MC, Chen HI, et al. De-regulated microRNAs in pediatric cancer stem cells target pathways involved in cell proliferation, cell cycle and development. PLoS ONE. 2013;8:e61622.

25. Gregory PA, Bert AG, Paterson EL, Barry SC, Tsykin A, Farshid $\mathrm{G}$, et al. The miR-200 family and miR-205 regulate epithelial to mesenchymal transition by targeting ZEB1 and SIP1. Nat Cell Biol. 2008;10:593-601.

26. Korpal M, Lee ES, Hu G, Kang Y. The miR-200 family inhibits epithelial-mesenchymal transition and cancer cell migration by direct targeting of E-cadherin transcriptional repressors ZEB1 and ZEB2. J Biol Chem. 2008;283:14910-4.

27. Wu H, Zhu S, Mo YY. Suppression of cell growth and invasion by miR-205 in breast cancer. Cell Res. 2009;19:439-48.

28. Zhang Z, Che X, Yang N, Bai Z, Wu Y, Zhao L, et al. miR-135b$5 p$ Promotes migration, invasion and EMT of pancreatic cancer cells by targeting NR3C2. Biomed Pharmacother. 2017;96:1341-8.

29. Rhodes LV, Martin EC, Segar HC, Miller DF, Buechlein A, Rusch DB, et al. Dual regulation by microRNA-200b-3p and microRNA-200b-5p in the inhibition of epithelial-tomesenchymal transition in triple-negative breast cancer. Oncotarget. 2015;6:16638-52.

30. Stinson S, Lackner MR, Adai AT, Yu N, Kim HJ, O'Brien C, et al. miR-221/222 targeting of trichorhinophalangeal 1 (TRPS1) promotes epithelial-to-mesenchymal transition in breast cancer. Sci Signal. 2011;4:pt5.

31. Eger A, Aigner K, Sonderegger S, Dampier B, Oehler S, Schreiber $\mathrm{M}$, et al. DeltaEF1 is a transcriptional repressor of E-cadherin and regulates epithelial plasticity in breast cancer cells. Oncogene. $2005 ; 24: 2375-85$. 